\title{
FutureJournal
}

\section{O Uso Criativo dos Mecanismos de Busca da Web 2.0 para Pesquisar Invenções e Criar Inovações Frugais}

\author{
Carlos Mamori Kono \\ Universidade Nove de Julho (UNINOVE), Brasil \\ carloskono@sistec.net.br \\ Luc Quoniam \\ Université Du Sud Toulon Var, França \\ mail@quoniam.info

\section{Leonel Cezar Rodrigues} \\ Universidade Nove de Julho (UNINOVE), Brasil \\ leonel@uninove.br \\ Hérmani Magalhães Olivense do Carmo \\ Universidade Federal de Alagoas (UFA), Brasil \\ hermani_record@hotmail.com
}

\section{RESUMO}

O crescimento exponencial da informação torna cada vez mais complexo o acesso ao acervo do conhecimento, pois exige instrumentos inovadores para processamento, armazenagem e recuperação da informação para serem aplicadas às necessidades das organizações. A Web 2.0 disponibiliza novas estratégias mercadológicas e novos processos de comunicação ao potencializar as possibilidades de trabalho coletivo e participativo, com a construção, disseminação e compartilhamento de informações apoiadas e intermediadas pelo computador. Neste estudo, de natureza qualitativa e nível descritivo, identificam-se as características de alguns mecanismos de busca da Web 2.0 para apropriação dos dados científicos e tecnológicos que possam ser utilizados para a pesquisa de invenções e criação de inovações frugais. Os resultados comprovaram que os recursos informacionais e cooperativos analisados permitem, potencialmente, auxiliar a criação de inovações frugais baseadas no efeito termoelétrico (efeito Peltier). Inovações baseadas nesse fenômeno devem tornar-se uma das alternativas mais promissoras para a promoção do desenvolvimento inclusivo das economias emergentes.

PALAVRAS-CHAVE: Ciência da informação. Web 2.0. Criatividade. Inovação frugal. 


\title{
FutureJournal
}

\section{The Creative Use of Search Engines Web 2.0 to Research Inventions and Create Frugal Innovations}

\begin{abstract}
The exponential growth of information has made the access to knowledge assets more complex, requiring organizations to develop innovative tools to process, store and retrieve information. The Web 2.0 system facilitates the development and sharing of information through the use of computers. As a result, the Web 2.0 offers new marketing strategies and communication processes, enhancing the prospects for collective and participatory work. This qualitative and descriptive study identifies the characteristics of some Web 2.0 search engines that can be used to collect scientific and technological data. The data retrieved by these engines can be used to create or further develop existing innovative frugal studies. The results show that the cooperative resources and information analyzed in this paper assist in the development of frugal innovations based on the thermoelectric effect (Peltier effect). As a conclusion, the innovations that are based on this phenomenon will become one of the most promising alternatives for promoting an inclusive development of emerging economies.
\end{abstract}

KEY-WORDS: Information science. Web 2.0. Creativity. Frugal innovation. 


\section{INTRODUÇÃO}

A competitividade crescente tem levado as empresas a buscar inovações nos seus modelos de negócios e a tendência atual é atingir os mercados emergentes, pouco explorados, mas com enorme potencial de consumo. Atender às demandas dos consumidores com recursos limitados, leva à criação de inovações frugais - produtos suficientemente bons com preços acessíveis (Zeschky, Winterhalter \& Gassman, 2014), especialmente desenvolvidos para alcançar um mercado potencial de quatro bilhões de pobres aspirantes (base da pirâmide), considerado um mercado de consumo gigantesco onde a escala é tudo (Prahalad \& Hart, 2002).

Criar inovação frugal significa voltar ao básico, desenvolver produtos baseados em arquiteturas disruptivas para atingir um grupo de clientes totalmente novo utilizando materiais e tecnologias já existentes (Zeschky et al., 2014, p. 21). e contribuindo para a sustentabilidade do planeta, com base em três premissas: economicamente sustentada no tempo, socialmente inclusiva e ambientalmente sustentável (Sachs, 2004), gerando eficiência econômica, equidade social e prudência ecológica (Strong, 1993).

A explosão informacional, significando o crescimento exponencial da informação e de seus registros, torna cada vez mais complexo o acesso ao acervo do conhecimento, especialmente na área da ciência e da tecnologia. É nesse cenário que surge o conceito de ciência da informação (CI) - um campo do conhecimento que envolve tanto a pesquisa científica quanto a prática profissional para a proposição de problemas e escolha de métodos para a sua solução (Bush, 1945). Ele compreende a efetiva comunicação da informação e objetos de informação, particularmente o registro do conhecimento para atender a dois mundos que interagem: o da tecnologia e o da humanidade (Saracevic, 1996).

A base da relação entre a CI e a ciência da computação (CC) é constatada pela aplicação de computadores e da computação na recuperação das informações dos produtos e serviços através de redes associadas. A CC trata de algoritmos que transformam informações, enquanto a CI trata da natureza da mesma informação e sua comunicação para ser utilizada pelos humanos. Ambas estão inter-relacionadas evoluindo para níveis diferentes de 
cooperação intelectual. Configuram, portanto, agendas básicas e aplicadas diferentes não exclusivas, mas complementares (Saracevic, 1996).

Nesse contexto, a Web 2.0 representa uma nova geração de serviços on line. O elevado número de dados e sua abrangência nas diversas áreas do conhecimento exigem a aplicação de mecanismos adequados para suportar os processos complexos de gestão da informação e do conhecimento. Utilizada como uma plataforma, disponibiliza novas estratégias mercadológicas e processos de comunicação, potencializando as possibilidades de trabalho coletivo e participativo, com a construção, disseminação e compartilhamento de informações apoiadas e intermediadas pelo computador (O'Reilly, 2005).

Esse cenário levou ao interesse de prospectar os instrumentos disponíveis na Web 2.0 para a recuperação de informações existentes na Internet que possam contribuir para a pesquisa de invenções e criação de inovações. A questão de pesquisa que se coloca para este estudo é: como usar de forma criativa os mecanismos de busca da Web 2.0 para pesquisar invenções e criar inovações frugais?

O objetivo é identificar as características dos mecanismos de busca, de uso livre e gratuito da Web 2.0 que permitem apropriar dados científicos e tecnológicos a serem utilizados para pesquisa de invenções e criação de inovações frugais. Os dados científicos estão relacionados com pesquisa e desenvolvimento e os dados tecnológicos com a sua aplicação para criação de invenções e inovações.

Neste estudo, será utilizado como referência o fenômeno gerador de invenção e de inovação baseado no efeito termoelétrico conhecido como efeito Peltier, que gera energia elétrica a partir da diferença de temperatura. Inovações baseadas nesse efeito devem tornar-se uma das alternativas mais promissoras para a geração de energia elétrica de baixo custo, em especial nas economias emergentes, que apresentam dificuldades para desenvolver infraestrutura básica para poder lutar contra a pobreza e promover o desenvolvimento inclusivo.

Foi adotada a abordagem qualitativa com enfoque descritivo e exploratório, relacionada com o vasto campo interdisplinar da CI, em especial com a aplicação dos recursos informacionais e cooperativos da Web 2.0. 
A estrutura deste estudo é composta da revisão bibliográfica dos conceitos teóricos que dão consistência aos temas abordados; dos métodos e técnicas de pesquisa empregados no delineamento desta pesquisa a fim de atingir o objetivo proposto; da apresentação e análise dos dados com as características de alguns mecanismos de busca e dos resultados práticos da sua aplicação para facilitar a pesquisa de invenções e criação de inovações; e finalmente, das considerações finais com as inferências em relação ao tema.

\section{REVISÃO BIBLIOGRÁFICA}

O estudo sobre o uso criativo dos mecanismos de busca da Web 2.0 para pesquisar invenções e criar inovações frugais levou à discusão de tópicos relacionados com CI, Web 2.0, invenção e inovação, inovação frugal e sustentabilidade, e competência e criatividade como suporte à análise das fontes de inovação.

\subsection{CIÊNCIA DA INFORMAÇÃO}

A explosão informacional, significando o crescimento exponencial da informação e de seus registros, torna cada vez mais complexo o acesso ao acervo do conhecimento, especialmente nas áreas da ciência e da tecnologia, considerado por Bush (1945, p. 10) como um dos mais importante insumos para atingir e sustentar o seu desenvolvimento. A importância estratégica da informação e do conheimento tem sido estendida a todos os campos, a todas as tarefas humanas e a todos os tipos de empreendimentos e alinhada, de forma mais consistente, nos conceitos de inteligência estratégica ou competitiva.

Esse é o campo de atuação da CI, definido como o estudo da forma como as pessoas criam, usam e comunicam informações (Becker, 1976, citado por Saracevic, 1996, p. 47) e que se interessa por situações de explosão da informação e por aplicações tecnológicas para a proposição de problemas e escolha de métodos para a sua solução. Abrange a efetiva comunicação da informação e de objetos de informação, particularmente o registro de conhecimento para atender a dois mundos que interagem: o da necessidade humana e social de uso da informação e o dos sistemas e 
tecnologias para atender àquela necessidade e para prover a efetiva organização e recuperação da informação (Saracevic, 1996, p. 47). O termo "recuperação de informação" engloba os aspectos intelectuais da descrição de informações e suas especificidades para a busca independente de sistemas, técnicas ou máquinas empregados para a operação (Mooers, 1951, p. 25).

A expansão da ciência e da tecnologia pode ser medida por meio do uso de diversas formas no campo da $\mathrm{CI}$ : qualitativas ou quantitativas ou uma combinação entre ambas (Vanti, 2002, p. 153). As técnicas quantitativas podem ser subdivididas nas seguintes disciplinas: 1) bibliometria, que estuda os aspectos quantitativos da produção, disseminação e uso da informação registrada; 2) cienciometria, que estuda os aspectos quantitativos da ciência como disciplina ou atividade econômica, aplicada no desenvolvimento de políticas científicas; 3) informetria, um subcampo emergente da CI (Wormell, 1998 , p. 2) que estuda os aspectos quantitativos da informação em qualquer formato, fora dos limites tanto da bibliometria quanto da cienciometria (Macia-Chapula, 1998, p. 134), com a compreensão de que a informetria compreende um campo mais amplo do que a cienciometria e a bibliometria (McGrath, 1989, citado por Macia-Chapula, 1998, p. 135).

Uma nova aplicação, também compreendida pela informetria, seria a webometria, que corresponde à aplicação de metodos informétricos desenvolvidos sobre o conteúdo e estruturas da Word Wide Web (Almind \& Ingwersen, 1997), com sobreposição, em alguns momentos, com a bibliometria e a cienciometria. Esta é uma área de estudos da cybermetrics, proposta por Almind e Ingwersen, e da internetometrics, proposta por Quoniam e Roustaing, que compreende: home pages pessoais, institucionais ou ad hoc; páginas que disponibilizam links, hyperlinks ou weblinks; e páginas que disponibilizam dados em forma de texto, som ou imagem (Vanti, 2002, p. 156-157).

Dessa forma, a informação passou a ter relevância para a sociedade, contribuindo para o surgimento e crescimento da $\mathrm{CI}$, por meio de estudos teóricos e experimentais sobre a natureza da informação: a estrutura do conhecimento e seus registros; seu uso e os usuários; a interação homem- 
computador; os atributos da informação (relevância, utilidade, obsolescência, etc.); a economia, o impacto e o valor da informação; dentre outros.

A base da relação entre a CI e a CC é constatada pela aplicação de computadores e da computação na criação dos bancos de dados; na recuperação das informações; e nos produtos, serviços e redes associadas. A CC trata de algoritmos que transformam informações, enquanto a CI trata da natureza da mesma informação e da sua comunicação para ser utilizada pelos humanos, ambas inter-relacionadas e evoluindo em direção a um nível diferente de cooperação intelectual. Não são, portanto, competitivas, mas complementares e conduzem para diferentes agendas básicas e aplicações (Saracevic, 1996, p. 50). Essa relação torna-se mais relevante quando se insere a Web no contexto do desenvolvimento das tecnologias da informação e comunicação e na busca da compreensão de como funcionam os mecanismos de divulgação e acesso às informações para fins acadêmicos. Dessa forma, o domínio de novas tecnologias é condição essencial para o progresso da pesquisa científica em CI.

\subsection{WEB 2.0}

No mundo atual, globalizado e totalmente conectado, a informação e o conhecimento estão dispersos por diversas áreas de estudo e a sua recuperação exige uma mudança de paradigma na visão dos processos relacionados com a ciência e tecnologia, pois envolve arcabouços teóricometodológicos complexos para a organização da informação, além da necessidade de instrumentos inovadores para processamento, armazenagem e recuperação da informação que possa permitir a sua posterior aplicação às necessidades das organizações.

Web 2.0 é o termo popular usado para identificar as tecnologias e aplicações avançadas da Internet incluindo blogs (que permitem expressar opiniões e comentários e manter conversações em nichos específicos), wikis (que permitem criação e a edição de páginas Web usando um browser Web), RSS - Really Simple Syndication (que permite divulgar conteúdo novo de forma rápida e precisa nos sites preferidos do usuário e disseminados via tecnologia denominada podcasting), mashups (que permitem consolidação 
de dados de duas ou mais fontes locais de Web para criar novas aplicações e redes sociais) (Lai \& Turban, 2008, p. 388).

As redes sociais são entendidas como uma estrutura na qual pessoas ou organizações interagem, colaboram e criam o próprio espaço (home pages), conectadas por um ou vários tipos de relações, compartilhando valores e objetivos comuns como fotos, blogs, ideias e links com outros locais da Web (Wikipedia, n.d.) como grupos de trabalho, negócios financeiros, amizade, parentesco, relações comerciais e associações profissionais, entre outros (Lai \& Turban, 2008, p. 390).

Assim, os usuários advindos dos mais diversos segmentos da sociedade deixam de ser predominantemente consumidores de informações para serem produtores de informação através de práticas colaborativas ou "arquitetura de participação" on line utilizando a Web 2.0 como uma plataforma (O'Reilly, 2005, p. 25).

A Web 2.0 representa uma nova geração de serviços on line pois potencializa as formas de publicação, organiza e compartilha informações; além de ampliar a interação entre os usuários. Utilizada como uma plataforma, disponibiliza novas estratégias mercadológicas e processos de comunicação, potencializando as possibilidades de trabalho coletivo e participativo, com a construção, disseminação e compartilhamento de informações apoiadas e intermediadas pelo computador.

No campo da CI, a Web 2.0 é uma fonte inesgotável de recursos para pesquisadores que buscam informações. Mas sua natureza dinâmica - seu acesso em tempo real, sua enorme massa de dados não padronizada, seu caráter mutante quanto ao conteúdo com a inclusão contínua de novas páginas e o desaparecimento de outras, e suas inconsistências nos serviços e mecanismos de busca - pode comprometer a busca e a recuperação de informação nessa grande base de dados (Vanti, 2002, p. 158). Dessa forma, tecnologias construídas no contexto da Web 2.0, como o Wikipedia, apresentam a oportunidade de compartilhar a informação e ampliar o processo interativo entre usuários e informações (Moura, 2009).

Com o advento da Web, começaram a surgir mecanismos específicos para pesquisa de suas páginas, que fornecem meios para localizar o que se busca entre as mais de um milhão de páginas HTML. Esses meios envolvem dois tipos básicos de mecanismos: 1) diretórios: são ferramentas genéricas 
ou temáticas que organizam os sites que compõem sua base de dados de forma hierárquica (categorias e subcategorias); 2) motores de busca: utilizam softwares que trabalham em paralelo para construir sua base de dados e permitir seu acesso pelos usuários. Seus resultados mostram uma lista de descrições de sites e links.

Existem diferenças essenciais entre os dois tipos básicos mencionados. Os diretórios têm bases de dados menores, mas contêm informações mais relevantes do que os motores de busca e são mais apropriados para busca por tópicos de interesse de grande número de pessoas. Já os motores de busca permitem a localização de qualquer tipo de informação, desde que exista na Internet, ordenando os itens recuperados pelo critério de relevância, cabendo ao usuário decidir quais são os de maior interesse. Os motores de busca procuram parcerias com diretórios, incluindo links com o objetivo de oferecer opções de maior seletividade de recursos (Cendon, 2001, p. 39).

\subsection{INVENÇÃO E INOVAÇÃO. INOVAÇÃO FRUGAL E SUSTENTABILIDADE}

A origem de novos produtos são as descobertas e as invenções resultantes de um processo de geração do conhecimento, economicamente irrelevante até ser convertida em inovação tecnológica, seja na forma de um novo produto, de uma nova técnica ou de um novo processo produtivo ou serviços.

A inovação é entendida como sinônimo para a produção, assimilação e exploração exitosa de uma novidade no contexto econômico e social, oferecendo soluções novas para problemas, possibilitando, dessa forma, encontrar necessidades tanto para o individuo como para a sociedade (European Commission, 1995, p. 1). É o processo de transformar oportunidades em novas ideias e colocá-las em práticas de uso extensivo (Freeman, 1982, citado por Plonski, 2005, p. 27).

Um requisito básico para um processo exitoso de inovação é criar uma inteligência virtual resultante de uma rede por onde fluem as informações e o conhecimento. Uma inovação, mais do que qualquer outra atividade econômica, depende do conhecimento. Isso significa que o conhecimento e, 
por extensão, a informação, passa a ter uma enorme implicação para a CI, assumindo papel central na ordem social e econômica.

A competitividade crescente tem levado as empresas a buscarem inovações nos modelos de negócios e a tendência tem sido atingir os mercados emergentes - ainda pouco explorados, mas com enorme potencial de consumo.

Nesse cenário, a inovação frugal configura o resultado de um processo que visa atender, em especial, às necessidades de mercados emergentes, fornecendo soluções funcionais com custo reduzido de produção, redesenhando produtos, reconfigurando as cadeias de valor e criando novos modelos de negócios (Bhatti, 2012, p. 18). Os empresários procuram criar mercados mais abrangentes, ao preencher os vazios institucionais, equacionar as limitações de recursos, minimizar os investimentos em P\&D e favorecer a rentabilidade de consumidores da base da pirâmide.

Inovações frugais notáveis têm explodido em economias emergentes, produzidas principalmente por pequenas empresas locais dos países emergentes. As multinacionais também começaram a envolver-se nesse mercado, enfrentando um desafio empresarial, tendo em vista que seus modelos de negócios e estruturas organizacionais são tradicionalmente concebidos para o desenvolvimento de produtos oferecidos para o topo da pirâmide econômica (Wikipedia, n.d.). Assim, dadas as vastas populações com poucos recursos, os empresários procuram ao máximo satisfazer as necessidades locais de forma prática e sustentável, historicamente negligenciadas pelas empresas tradicionais (Bhatti, 2012, p. 18), almejando colocar, de forma apropriada, adaptável, disponível e acessível, produtos e serviços que contribuem para a sustentabilidade do planeta, com base em três premissas: economicamente sustentadas no tempo, socialmente inclusivas e benignas do ponto de vista ambiental (Sachs, 2010, p. 30) e que geram eficiência econômica, equidade social e prudência ecológica (Strong, 1993, p. 1).

Oferecer soluções sustentáveis significa vencer os desafios que envolvem a fome no mundo, a pobreza, a injustiça social e a falta de recursos que afetam bilhões de pessoas (Basu, Banerjee \& Sweeny, 2013, p. 64). Em contraste com o custo de inovações adequadas, as inovações frugais 
constituem novidades tanto na perspectiva tecnológica quanto de mercado. Embora tecnologias existentes sejam empregadas na maioria dos casos, as inovações frugais são tipicamente construídas sobre novas arquiteturas de produtos que permitem novas aplicações a preços muito mais baixos que as soluções existentes (Zeschky et al., 2014, p. 23).

As inovações frugais têm surgido na área de veículos, celulares e eletrodomésticos e são caracterizadas pela supressão de funções não essenciais, redução do consumo de recursos não renováveis, consumo reduzido de energia elétrica, entre outras, buscando alinhar seus custos e necessidades com as carências existentes na base da pirâmide (Wikipedia, n.d.).

\subsection{COMPETÊNCIA E CRIATIVIDADE COMO SUPORTE À ANÁLISE DAS FONTES DE INOVAÇÃO}

As empresas devem ser vistas não apenas como um portfólio de produtos ou serviços, mas também como um portfólio de competências, entendidas como a capacidade de combinar, misturar e integrar recursos organizacionais que favoreçam o desenvolvimento de produtos e serviços, garantindo dessa forma a competitividade da empresa. Essa situação associa a noção de competência a verbos como "integrar saberes múltiplos e complexos, saber aprender, saber engajar-se, assumir responsabilidades, ter visão estratégica" (Fleury \& Fleury, 2001, p. 187).

O termo "competência" no âmbito da tomada de decisão e gestão de recursos tem sido tratado exaustivamente por pesquisadores da área de estratégia, inteligência competitiva e de inovação. Mas existem outros atributos relevantes que Ruas (2005, p. 2) denomina "competências voláteis", caracterizadas por sua intangibilidade. São competências pouco exploradas no mundo dos negócios, como abstração, percepção, improvisação, negociação e criatividade, embora muito utilizadas no cotidiano dos mais diversos campos profissionais. Dentre essas, a de maior relevância é sem dúvida nenhuma a criatividade, que está associada com ideia criativa, invenção ou descoberta e pode transformar-se em inovação. 
Criatividade é pensar em algo original enquanto inventar é implantar uma ideia criativa, sendo a inovação sua execução (Valentim, 2008, p. 4). Os termos estão intrinsecamente ligados, um pela geração de ideia, outro por sua aplicação prática. No sentido inverso, a inovação torna-se, também, um processo essencial para promoção da criatividade através da qual a contribuição criativa é refinada e divulgada para o mundo externo, trazendo uma nova solução para uma ideia ainda em uso. Amabile, Conti, Coon, Lazenby e Herron (1996, p. 1155) associam a criatividade à produção de ideias novas e úteis em qualquer domínio e a inovação a uma bem-sucedida implementação de ideias criativas dentro de uma organização. Assim, nenhuma inovação é possível sem os processos criativos para identificar problemas e oportunidades, reunir informação, gerar novas ideias e explorálas.

A criatividade e a inovação constituem elementos-chave para o aprimoramento organizacional (Feldman, Ruthes \& Cunha, 2008, p. 239). Essa combinação tem despertado crescente interesse das organizações devido à necessidade de rápida adaptação às mudanças, impulsionadas por meio de práticas inovadoras de negócios, processos, produtos e serviços. Evidências empíricas de que a criatividade está ligada à inovação podem ser encontradas nos estudos de Blau e McKinley (1979) no desenvolvimento de projeto em uma empresa de arquitetura, de Bharadwaj e Menon (2000), que identificaram contribuições da criatividade nas atividades individuais e organizacionais para o desempenho da inovação, e de Soo, DeVinney, Midgley e Deering (2002), que identificaram a criatividade como o principal fator para criação de novos conhecimentos e inovações para solução de problemas (Puccio \& Cabra, 2010, p. 147). Alguns portais da Web 2.0 acessados neste estudo discutem a relação da criatividade com a inovação.

Nessa relação da competência, criatividade e inovação, fica a questão de como e onde buscar as fontes de informações e de conhecimento que possam contribuir para os processos de inovação. Atualmente, a maior fonte de informações é encontrada na Web, uma plataforma que favorece a colaboração e o compartilhamento de conteúdo, envolvendo qualquer tipo de usuário (pessoa ou empresa) e permitindo gerar maior valor para a criação de novos produtos e serviços. Grandes grupos ou redes de pessoas são 
muitas vezes mais inteligentes do que uma pequena elite. E podem ser mais efetivos para a solução de problemas, promoção da inovação, tomada de decisões mais sábias e até para previsão do futuro. O princípio subjacente por trás do sucesso da Web 2.0 é o uso inteligente da inteligência coletiva dos membros de determinado grupo e a transformação da Web em um cérebro global (Lai \& Turban, 2008, p. 394).

Pelo fato de os mecanismos de busca da Web usarem estrutura de links, os blogs - que são os mais produtivos e atualizados usuários de links exercem papel relevante nos resultados dos mecanismos de busca e seu poder passa a ser relevante no processo da blogosfera, entendido com um novo meio de comunicação entre usuários, não apenas para obter fácil acesso aos comentários individuais de uma página, mas também por aceitar criação ou inclusão de links e comentários na própria página (O 'Reilly, 2005, p. 7).

\section{MÉTOdOS E TÉCNICAS DE PESQUISA}

\subsection{DESENHO DE PESQUISA}

No cenário que envolve este estudo, o que se pretende é conhecer, explicitar e explorar os mecanismos de busca da Web 2.0 que permitam acesso à base de dados com a finalidade de buscar informações sobre o efeito termoelétrico que possam contribuir para a pesquisa de invenções, criação de inovações e campo da CI.

A questão de pesquisa neste trabalho propõe a exploração de informações dispersas em diversas plataformas de informações, principalmente no Google Scholar, no Wikipedia e nos diversos sites e blogs da Web 2.0.

O desenho metodológico da presente pesquisa empírica é de natureza qualitativa buscando obter dados mediante contato direto e interativo de seus autores com a situação objeto de estudo (mecanismos de busca da Web 2.0), a fim de procurar entender os fenômenos e avaliar sua contribuição para o objetivo do estudo (pesquisa de invenções e criação de inovações), sem aplicação de instrumental estatístico para análise dos dados. Será conduzido em nível descritivo quanto aos fenômenos pesquisados, apenas mostrando a 
realidade como ela é, embora, posteriormente, os resultados possam ser usados para testar e formular teorias (Eisenhardt, 1989).

Este estudo será tratado por meio de estudo de caso, apropriado nas situações em que a questão de pesquisa é uma interrogação do tipo "como" ou "por que", preferido nas propostas de investigação em profundidade de um fenômeno atual inserido no contexto da vida real (Yin, 2010).

O atendimento aos objetivos desejados para este estudo - identificar as características dos mecanismos de busca da Web 2.0 de uso livre e gratuito e verificar como esses mecanismos podem facilitar a interação dos dados no campo da CI - levou à decisão de não se elaborar apenas um inventário dos mecanismos de busca da Web 2.0, mas incluir esse objetivo num cenário mais abrangente, abarcando procedimentos relacionados com a gestão de informação e do conhecimento e a gestão da inovação.

Para a aplicação prática dos mecanismos de busca, foram adotados como termo de pesquisa "efeito Peltier", pois esse é o fenômeno a partir do qual de pretendeu pesquisar invenções e avaliar a possibilidade de criar inovações. No caso de "inovação", buscou-se também a combinação com "criatividade", para entender o processo de geração de ideias, e "inovação frugal", para verificar o entendimento do tema.

\subsection{O EFEITO TERMOELÉTRICO. EFEITO PELTIER}

Devido às inúmeras características físicas e funcionais que podem caracterizar uma inovação frugal, neste estudo, utilizou-se a tecnologia baseada no fenômeno termoelétrico conhecido como efeito Peltier como fator gerador para inovação de produto.

O efeito termoelétrico é a conversão direta da diferença de temperatura em tensão elétrica e vice-versa. Um dispositivo termoelétrico cria uma tensão elétrica quando há uma diferença de temperatura entre seus extremos (efeito Peltier). Quando acontece o contrário, ou seja, é aplicada uma tensão elétrica, o efeito termoeletrico cria uma diferença de temperatura (efeito Seeback). Em muitos livros e artigos, o efeito termoelétrico pode ser chamado efeito Peltier-Seebeck. Essa separação provém de descobertas 
independentes do físico francês Jean Charles Athanase Peltier e do físico estônio-alemão Thomas Johann Seebeck (Wikipedia, n.d.).

A aplicação prática do efeito Peltier está na geração de energia por meio do processo de troca de calor, que pode contribuir para a inclusão de comunidades que não contam com energia elétrica para atender a suas necessidades cotidianas.

\section{APRESENTAÇÃO E ANÁLISE DOS DADOS}

Neste tópico são apresentados os mecanismos de busca, de uso livre e gratuito identificados na Web 2.0, que permitem apropriar dados científicos e tecnológicos para serem utilizados na pesquisa de invenções e criação de inovações frugais.

A pesquisa de invenção e criação de inovação, está relacionada com uma efetiva gestão da inovação, que inclui tanto os procedimentos e as competências organizacionais como a elaboração de projetos de inovações. Significa uma gestão da inovação mais sistemática e mais objetiva ao: 1) buscar, avaliar e organizar informações que possam constituir um inventário de tecnologias de invenções e de criação de inovações; 2) escolher a lógica de como a inovação pode criar valor; e 3) oferecer opções para criação de possíveis inovações frugais.

Dessa forma, os mecanismos identificados estão distribuídos nos seguintes tópicos principais: 1) software Zotero, gerenciador de referências bibliográficas; 2) Google Acadêmico, para pesquisa de literatura acadêmica; 3) Wikipedia, como thesaurus para inovação. Links cooperativos; 4) patentes como fonte de inovações. Base de patentes. Instrumentos de análise; e 5) Um método para a solução criativa de problemas: matriz TRIZ.

\subsection{SOFTWARE ZOTERO, GERENCIADOR DE REFERÊNCIAS BIBLIOGRÁFICAS}

A elaboração da fundamentação teórica dos temas principais deve incluir a preocupação com o armazenamento e controle de todos os dados que passam a fazer parte do estudo, tanto os qualitativos quanto os 
quantitativos. Sendo assim, o software gerenciador de referências bibliográficas Zotero torna-se relevante nesta etapa do estudo.

O Zotero (ZO) é um software que permite a importação e o armazenamento das referências e dos links dos artigos de diversas bases de dados da literatura técnico-centífica da Web 2.0, para uma base individual criada pelo próprio pesquisador e que pode ser compartilhada com outros usuários. Grosso modo, substitui o antigo ficheiro de artigos, autores e citações usados historicamente em uma pesquisa. Opera com o navegador Mozilla Firefox, software de uso também livre e gratuito.

Trata-se de um software para gestão de referências bibliográficas, constituindo um importante recurso para coletar, organizar, controlar e compartilhar todas as fontes extraidas das bases de dados arquivos PDF, imagens, arquivos de áudio e vídeos, fotos de páginas da Web, entre outros. Esse software também automatiza as referências bibliográficas e permite sua integração com o processador de texto MS-Word, por meio da opção "Suplementos". As referências de citações e paráfrases das publicações são selecionadas para formar a bibliografia de forma simples e automática, podendo utilizar os formatos de citações e referências mais comumente adotados como os padrões APA, ABNT, Chicago, entre outros.

Para este estudo, o termos para busca foram "thermoeletric effect", "Peltier effect" e "frugal innovation". Todos os procedimentos de pesquisa foram realizados por meio do ZO, que permitiu maior agilidade e eficiência nas pesquisas e armazenamento dos artigos. Podem ser citadas as seguintes vantagens e facilidades de uso: 1) pesquisa e visualização de documentos com extensões .html, .pdf, .doc); 2) avaliação da pertinência da informação ao objetivo da pesquisa; 3 ) organização dos documentos pertinentes em pastas específicas; 4) facilidade e rapidez no processo de coleta de informações; 5) classificação e armazenamento dos documentos respeitando suas extensões; 6) seleção de parte dos textos coletados considerados relevantes para o processo de criação de inovações (notes); 7) compartilhamento dessa base criada com usuários autorizados para atividades cooperativas, visando consolidar informações e conhecimentos.

O ZO facilita o gerenciamento dos documentos, tornando transparente as atividades realizadas pelos coletores e apoiando a 
sistematização das atividades realizadas na fase de coleta e análise nos acessos ao Wikipedia, Google, Google Scholar/Books/Patents, YouTube e revistas.

\subsection{O GOOGLE ACADÊMICO COMO BASE PARA PESQUISA BIBLIOGRÁFICA}

A base de dados do Google Acadêmico (GA) inclui a literatura acadêmica constituída de teses, livros, resumos e artigos de editoras acadêmicas, bibliotecas de pré-publicações, universidades e demais organizações acadêmicas. É de extrema relevância para o processo de popularização e democratização da informação acadêmica disponível na Web.

O software Harzing's Publish or Perish

(PoP)

(http://www.harzing.com/pop.htm) permite as buscas na base de dados do GA e calcula alguns índices científicos de forma muito mais prática e rápida do que o ISI ou o Scopus, que utilizam base de dados própria, mas menos abrangente do que a do GA.

Resultados da pesquisa realizada com o termo "Peltier Effect" no título, no período de publicação de 2005 a 2014. Total de artigos: 100; artigos/autor: 42.23; total de citações de todos artigos: 611; citações por ano: 61.10; citações/autor/ano: 20.69

Como exemplo, no Quadro 1, mostram-se os dados relacionados com os cinco artigos mais citados na base de dados do GA.

\begin{tabular}{|c|c|c|c|c|c|c|}
\hline $\begin{array}{l}\text { Numerocit } \\
\text { ações }\end{array}$ & $\begin{array}{l}\text { Cit/ } \\
\text { ano }\end{array}$ & Autores & Título & Ano & Publicação & Fonte \\
\hline 75 & 25.00 & $\begin{array}{l}\text { J Flipse, FL } \\
\text { Bakker, A } \\
\text { Slachter, FK } \\
\text { Dejene... }\end{array}$ & $\begin{array}{l}\text { Direct observation of the spin- } \\
\text { dependent Peltier effect }\end{array}$ & 2012 & Nature ... & $\begin{array}{l}\text { nature. } \\
\text { com }\end{array}$ \\
\hline 67 & 7.44 & $\begin{array}{l}\text { L Gravier, S } \\
\text { Serrano- } \\
\text { Guisan, } \\
\text { F Reuse, JP } \\
\text { Ansermet }\end{array}$ & $\begin{array}{l}\text { Spin-dependent Peltier effect of } \\
\text { perpendicular currents in } \\
\text { multilayered nanowires }\end{array}$ & 2006 & $\begin{array}{l}\text { Physical Review } \\
\text { B }\end{array}$ & APS \\
\hline 60 & 7.50 & $\begin{array}{l}\text { P DiCarlo, J } \\
\text { Megerman }\end{array}$ & $\begin{array}{l}\text { Ablation probe with Peltier effect } \\
\text { thermal control }\end{array}$ & 2007 & $\begin{array}{l}\text { US Patent } \\
7,238,184\end{array}$ & $\begin{array}{l}\text { Google } \\
\text { Patents }\end{array}$ \\
\hline 59 & 5.90 & $\begin{array}{l}\text { D Astrain, JG } \\
\text { Vián, J Albizua }\end{array}$ & $\begin{array}{l}\text { Computational model for } \\
\text { refrigerators based on Peltier effect } \\
\text { application }\end{array}$ & 2005 & $\begin{array}{l}\text { Applied Thermal } \\
\text { Engineering }\end{array}$ & Elsevier \\
\hline 37 & 4.11 & $\begin{array}{l}\text { A Kyarad, } \mathrm{H} \\
\text { Lengfellner }\end{array}$ & $\begin{array}{l}\text { Computational model for } \\
\text { refrigerators based on Peltier effect } \\
\text { application }\end{array}$ & 2006 & $\begin{array}{l}\text { Applied physics } \\
\text { letters }\end{array}$ & $\begin{array}{l}\text { ieeexplore } \\
\text { ieee.org }\end{array}$ \\
\hline
\end{tabular}

\section{Quadro 1: Pesquisa Harzing}

Fonte: Harzing's Publisher or Perish (2014) 
Analisando-se os campos "Publicação" e "Fonte" no Quadro 1, percebe-se que alguns temas estão relacionados com a ciência e outros com a tecnologia, situação que pode ser evitada por pesquisa por publicação a fim de selecionar apenas artigos que interessem a determinada pesquisa. A transferência dos resultados para uma planilha Excell permite elaborar uma análise bibliométrica.

\subsection{A WIKIPEDIA COMO THESAURUS PARA INOVAÇÃO}

Um thesaurus caracteriza uma obra de referência que lista palavras agrupadas de acordo com a semelhança de significado (contendo sinônimos e antônimos), em contraste com um dicionário, que estabelece definições de palavras e geralmente as apresenta em ordem alfabética. Assim, a Wikipedia ajuda o interessado a encontrar a palavra, ou palavras, através da qual uma ideia pode ser mais bem e adequadamente expressa.

A Wikipedia, considerada pelo próprio portal, como a "enciclopedia livre que todos podem editar" (Wikipedia, n.d.), disponibiliza uma série de páginas e links acessáveis por qualquer usuário para explicar sua filosofia; portais temáticos, portais comunitários e ferramentas que permitem colocar as páginas em categorias e facilita a navegação dos usuários entre as páginas relacionadas com determinado tópico. As categorias podem tornar-se subcategorias de outras categorias que estão ligadas através de uma estrutura em árvore, auxiliando os leitores a encontrarem artigos sobre determinados temas utilizando a navegação pelas páginas.

As pesquisas relacionadas com diversos temas e subtemas permitem avaliar o potencial de informação proporcionado pela Wikipedia. Para este estudo, alguns portais, a seguir mencionados, foram utilizados como fontes de informação com combinação de termos ou categorias/subcategorias para a busca relacionada a criatividade, inovação, inovação frugal, efeito termoelétrico e efeito Peltier.

Os procedimentos diretamente relacionados com a Wikipedia tratam de mecanismos que acessam ou criam outras bases de dados, por meio de procedimentos que acompanham a filosofia de compartilhamento da informação, construídas por processos interativos entre usuários e 
informações. Na relação com dados da Wikipedia, o pesquisador não é um agente passivo que coleta as informações, mas um agente ativo e participativo que fornece e discute conhecimento, interagindo com grupos existentes ou construindo novos. Dessa forma, cria-se uma efetiva rede de informação e conhecimento colaborativa. A seguir são apresentados alguns links cooperativos.

\subsubsection{Categorização: criatividade, inovação e termoeletricidade}

\subsubsection{Criatividade (Créativité)}

(http://fr.wikipedia.org/w/index.php?title=Cat\%C3\%A9gorie:Cr\%C3 $\%$ A9ativit\%C3\%A9\&oldid $=88447722$ )

Link cooperativo em língua francesa, discutindo (Discussion) a criatividade e sua aplicação em diversas categorias (Catégorie) e subcategorias. Contém links para outros diversos assuntos.

Páginas disponíveis encontradas na pesquisa: conception de produit, creative problem solving, innovation, innovation frugale, TRIZ.

Existe link cooperativo análogo em língua inglesa, discutindo(Talk) a criatividade e sua aplicação em diversas categorias (Category) e subcategorias.

\subsubsection{Gestão da criatividade (Management de la créativité)}

(http://fr.wikipedia.org/w/index.php?title=Management_de_la_cr\% C3\%A9ativit\%C3\%A9\&oldid=89093761)

Portal temático sobre a gestão da criatividade, disponibilizando links para outros temas, trata de assuntos como desafios da gestão de criatividade, inovação em equipe, como ter personalidade criativa e ferramentas e metodologias de ajuda à criatividade.

\subsubsection{Inovação (Innovation)}

(http://en.wikipedia.org/wiki/Category:Innovation) 
Portal temático sobre a inovação, com 16 subcategorias.

Páginas disponíveis encontradas na pesquisa: innnovation, communities of innovation, innovation management, novelty, social innovation, technological innovation system.

\subsubsection{Termoeletricidade (Thermoeletricity)}

(https://en.wikipedia.org/wiki/Category:Thermoelectricity)

Portal com 19 subcategorias

Páginas disponíveis encontradas na pesquisa: Seeback coefficient, thermoelectric effect, thermoelectric generator, thermoelectric materials

\subsubsection{DBpedia (http://dbpedia.org/About) e DBpedia Spotlight} (https://github.com/dbpedia-spotlight/dbpedia-spotlight/wiki

O objetivo do DBpedia (Data Base Wikipedia) é formar uma comunidade para extrair conteúdos estruturados (conteúdos "processáveis" por máquinas que conseguem extrair informações e conhecimentos de maneira rápida e simples) da Wikipedia, disponibilizando-os como um banco de dados para pesquisadores ou empresas. Significa que o DBpedia aproveita a vasta gama de informações disponíveis nas mais de 250 línguas da Wikipedia para extrair, converter e criar conjuntos de dados estruturados.

Interfaces e serviços são disponibilizados para os usuários interagirem com seus serviços, compartilharem as fontes de informação da Wikipedia e ampliarem o conhecimento humano sobre determinado tema. Dessa forma, o DBpedia incentiva a comunidade de pesquisadores a constituir grupos de trabalho cooperativos alimentadores de informações. Cria-se, então, uma gigantesca base de dados sobre os mais variados temas e nas mais variadas línguas, que fica disponível para as empresas.

O DBpedia Spotlight, por sua vez, é uma ferramenta que permite a detecção e a identificação dos recursos disponíveis na Wikipedia e DBpedia para determinado texto, estabelecendo o link das palavras que fazem parte da Wikipedia. Exemplo: a inclusão do "abstract" deste estudo no Dbpedia Spotlight mostra os links associados a algumas palavras do texto: 
"The exponential growth of information becomes more complex their access to knowledge assets, requiring innovative tools for processing, storage and retrieval of information to attend the needs of organizations. Web 2.0 offers new marketing strategies and new communication processes to enhance the possibilities for collective and participatory work with the construction, dissemination and information sharing supported and mediated by the computer. Results showed that the informational and cooperative resources analyzed allow potentially assist in building the frugal innovations on thermoelectric based effect (Peltier effect). Innovations based on this phenomenon will become one of the most promising alternatives for promoting inclusive development of emerging economies."

Os termos em grifo mostram os links da Web.

\subsubsection{Carrot2 Clustering Engines}

(http://search.carrot2.org/stable/search)

Clustering Engines são mecanismos utilizados para analisar as pesquisas na Internet, categoriza os resultados ou trata-os como coleções de documentos. A informação é classificada em clusters e outras subcategorias para facilitar a análise dos dados para uma pesquisa.

Recomenda-se não filtrar os resultados de uma busca, porque isso reduz a oportunidade de criar inovações. O filtro, quando direcionado pelos "preconceitos" do usuário, condiciona a análise para os itens que o indivíduo já conhece, limitando, dessa forma, eventuais possibilidades de analisar outras possíveis inovações.

OCarrot2 é uma fonte de resultados de pesquisa por categorias temáticas, podendo agrupar automaticamente pequenas coleções de documentos como artigos, imagens, vídeos, fotos, etc.

Como exemplo, a pesquisa por "Peltier Effect", gerou 97 tópicos (para um total de 221.000) nas seguintes categorias principais: definition of Peltier Effect, Peltier cooler, Thermoelectric generator, thermoelectric devices, Peltier elements, etc. 


\subsubsection{Clusty (http://clusty.com)}

Mecanismo de busca na base de dados do Wikipedia semelhante ao Carrot2, com levantamento das informações por clouds. Cada cloud possui subtemas. A opção All clouds permite ampliar os campos de busca e análises.

Ao pesquisar-se por "Peltier effect", foram mostrados 25 tópicos agrupados por thermoelectric (7 tópicos), Leonard Peltier (7), Electronic, Controller (4) e Other Topic (7).

\subsubsection{Cluuz Search (www.cluuz.com/)}

Outro mecanismo de busca para mapeamento de pessoas que estudam determinados assuntos.

A pesquisa do tema "Peltier effect" encontrou 10 referências de um total de 449.000 resultados, apresentados por temas ou entidades/autores mais citados, permitindo, inclusive, a associação entre esses temas e as entidades, com possibilidades de uma apresentação gráfica das relações entre os temas e os autores.

Permite, dessa forma, compartilhamento e colaboração entre usuários ligados ao tema.

\subsubsection{Google trends}

(https://www.google.com.br/trends/explore\#cmpt=q)

É uma ferramenta que mostra, a partir das pesquisas realizadas no Google, os temas (categorias) mais populares acessados recentemente, utilizando gráfico de frequência em determinado período (tempo) e lugar do mundo (paises, regiões, cidades e linguagem).

Por exemplo, um dos temas mais pesquisados em 2014 foi a busca de informação sobre sustentabilidade e empreendedorismo.

\subsection{PATENTES COMO FONTE DE INOVAÇÕES}


As patentes apresentam as informações mais recentes de um setor tecnológico, tecnologia e produto; direcionam os esforços de P\&D no sentido de apontar novos caminhos, evitando a duplicação de esforços; e indicam investimentos no que pode ser efetivamente novo, tendo em vista a disponibilidade de informações técnicas bem antes do que outras fontes (Ferreira, Guimarães \& Contador, 2009). Os dados de patentes não são estáticos. Patentes citam outras patentes, e essas ligações proporcionam uma visão dinâmica dos antecedentes tecnológicos e descendentes de invenções patenteadas. Ao longo do tempo, acumulam citações que permitem aos pesquisadores identificar patentes mais citadas que tiveram impacto sobre as invenções subsequentes.

O rápido crescimento do número de registros de patentes tem exigido o desenvolvimento de ferramentas e de processos complexos de análise de patentes, que são importantes para apontar as tendências tecnológicas futuras, as estratégias tecnológicas, as preocupações com a violação de patentes e a competência para avaliar patentes promissoras (Abbas, Zhang \& Khan, 2014).

\subsubsection{As bases de patentes EPO, WIPO, Google Patents, USPTO}

Diversas base de dados de patentes podem ser acessadas na Internet de forma gratuita.

O European Pattent Office (EPO) administra, através do Espacenet, as patentes depositadas em cerca de 40 países-membros da Organização da Patente Europeia (EPC). Disponibiliza mais de 90 milhões de documentos com informações de invenções e de desenvolvimentos técnicos desde 1978. Permite a busca por título, resumo, número de publicação, aplicação, data de publicação, nome dos inventores e aplicadores e funcionalidades ou aplicabilidade das invenções.

O World Intellectual Property Organization (WIPO) conta com 146 países signatários (até setembro de 2012). O programa de acesso a sua base de dados de patentes, o PatentScope, permite acesso a patentes, marcas, desenhos industriais, direitos autorais e recursos, programas de atividades, 
notícias e eventos, além de links para um total de cerca de 30 milhões de documentos.

O Google Patents permite a indexação para patentes e pedidos de patentes da United States Patent and Trademark Office (USPTO), EPO e WIPO. Seus documentos incluem a coleção inteira de patentes concedidas e pedidos de patentes publicados a partir de cada banco de dados (que pertencem ao domínio público). Os documentos de patentes dos EUA registram dados desde o ano de 1790, algumas destruídas em um incêndio em 1836, mas houve melhor gerenciamento com a criação da USPTO em 1975; a EPO e WIPO, desde 1978.

Como exemplo, na Tabela 1, é possível avaliar as bases de dados administradas pela Espacenet, WIPO e GooglePatents.

Tabela 1: Pesquisa nas bases de Patentes

\begin{tabular}{l|c|c|c|c}
\hline Palavras de busca & Espacenet & PatentScope & USPTO & $\begin{array}{c}\text { Google } \\
\text { Patents }\end{array}$ \\
\hline thermoelectric effect & 2.721 & 1.905 & 513 & 537.000 \\
\hline $\begin{array}{l}\text { thermoelectric Peltier } \\
\text { "Peltier effect" }\end{array}$ & 121 & 1.083 & 311 & 444.000 \\
\hline $\begin{array}{l}\text { "thermoelectric" "Peltier" } \\
\text { "frugal" }\end{array}$ & 0 & 977 & 3.234 & 16.900 \\
\hline
\end{tabular}

Fonte: Espacenet (n.d.), Patentscope (n.d.), GooglePatents (n.d.)

O universo do Google Patents é de cerca de oito milhões de patentes incluindo pedidos e concessão. Em pesquisa nesse universo, o termo "frugal" em inglês teve o sentido de "moderado", levando a ignorar os dados para uma eventual análise. Em princípio, as patentes consideradas interessantes para análise desta pesquisa, foram obtidas por meio do termo "Peltier Effect", embora análises mais refinadas possam eliminar algumas opções que não estão relacionadas com inovação frugal.

\subsubsection{Instrumentos para análise de patentes}

\subsubsection{Intellixir (http://www.intellixir.com/)}


É uma principais ferramentas para analisar patentes e literatura científica para pesquisa visando a inovação e inteligência competitiva, muito versátil e relevante para análise bibliométrica em torno de determinado tema e para visualização de redes colaborativas de empresas e pesquisadores. Possibilita ver a evolução de determinada tecnologia em um domínio particular de conhecimento permitindo descobrir tecnologias emergentes, monitorar o estado-da-arte de uma tecnologia antes do planejamento ou lançamento de um novo produto e expandir a informação e o conhecimento no campo da CI.

È utilizada por grandes organizações públicas e privadas que operam em áreas relacionadas com ciência e tecnologia, química, farmacêutica, aeroespaço, eletrônica, entre outras. Como exemplo, foi possível buscar 102 textos sobre "Peltier", 467 sobre "innovation management de projets innovants", 161 em "créativité em éco-innovation", 16 sobre "thermoélectricité thermodynamique et apllications" e 1 sobre "innovation frugale". É uma fonte relevante de dados para criação de inovações.

\subsubsection{Open patent services (OPS) \\ (http://www.epo.org/searching/free/ops.html)}

É um instrumento de consultas automáticas, fornecido pela EPO como um serviço de entrega de dados de patentes. Permite aos usuários registrados o acesso aos dados brutos do EPO através de uma interface XML padronizada. Permite extrair dados da EPO, como o estado bibliográfico legal, o texto completo, os dados de Registro Europeu e imagens. Outras características do OPS: permite o acesso aos dados de patentes mundiais da EPO, enriquece as próprias aplicações de software e busca clientes com necessidades específicas, integrando seus dados com aqueles disponibilizados através do OPS.

\subsubsection{Aulive (http://www.aulive.com/)}

É considerado um método prático e fácil para contribuir para a inovação de produtos e processos, baseia-se em quatro ferramentas práticas 
relacionadas com planejamento estratégico, definição de problemas e geração de ideias:

1. Patent Inspiration: permite o acesso ao conhecimento por meio do banco de dados global de patentes com ênfase na inovação tecnológica, no mercado, no mapeamento da tecnologia e na forma como os problemas estão sendo solucionados pelas universidades, empresas e centros de pesquisa;

2. More Inspiration: oferece uma coleção de grandes inovações, com o banco de dados atualizado diariamente de forma a oferecer sempre novas doses de inspiração. Procura classificar todo novo produto ou processo da empresa de acordo com as tendências inovadoras, com as trajetórias tecnológicas e com produtos e processos que desempenham as mesmas funções;

3. Production Inspiration: organiza o conhecimento de acordo com sua função, de forma a sugerir novas formas de resolver determinado problema, descobrir quais tecnologias estão competindo no momento e encontrar novos mercados para as tecnologias.

4. Test My Creativity: oferece um perfil do nível de criatividade pessoal em oito áreas diferentes por meio de pontuação posteriormente comparada com a pontuação média global.

\subsection{UM MÉTODO PARA A SOLUÇÃO CRIATIVA DE PROBLEMAS MATRIZ TRIZ (www.triz40.com)}

Uma parcela crescente das empresas vem procurando aumentar a competitividade melhorando não somente a prática empresarial, mas também a pesquisa na área de desenvolvimento de produtos, tradicionalmente desdobrada na necessidade da redução do tempo de desenvolvimento do produto, do aumento da qualidade e da redução do custo dos produtos em função de seus ciclos de vida.

Uma abordagem diferenciada para a solução criativa de problema é proposta pela matriz TRIZ (MT) - sigla russa para Teoria da Solução Inventiva de Problemas e um de seus métodos nomeado Método dos Princípios Inventivos (MPI). Desenvolvida por Altshuller (1984), seu objetivo principal é aplicar suas estratégias e ferramentas para a geração de ideias criativas a 
fim de melhorar e/ou criar novos produtos e buscar a formulação e a solução de problemas por meio de sistemas técnicos (ST) sob forma de 40 princípios inventivos, que se mostraram responsáveis por praticamente 40 mil patentes, que apresentaram soluções verdadeiramente inovadoras.

No caso deste estudo, encontrar uma aplicação do efeito Peltier, pesquisar invenções e desenvolver uma inovação frugal constitui um processo em que o alinhamento da criatividade com a competência muito se relaciona com a proposta da TRIZ, com a qual se busca: 1) identificar o nome do ST, ou seja, inovação frugal (IF); 2) identificar a(s) função(ões) da IF a partir do efeito termoelétrico e efeito Peltier; 3) identificar os principais elementos da IF e respectivas funções; 4) descrever o funcionamento da IF; 5) definir os recursos necessários para a funcionalidade da inovação frugal (componentes, funções, indicador Peltier de calor necessário, energia gerada, etc.); e 6) formular o resultado final da IF.

Assim, o método de desenvolvimento de IF a partir da proposta da TRIZ é útil para resolver os problemas relacionados com a melhoria de produtos e o desenvolvimento de novos. No caso específico deste estudo relacionado com o efeito termoelétrico para criação de IF, os PIs devem ser reavaliados e revisados de modo a considerar as informações e o conhecimento obtidos pelos diversos mecanismos de busca anteriormente mencionados.

Os mecanismos de busca identificados neste estudo estão agrupados no Quadro 2, em que se apresenta a correlação das características dos mecanismos de busca com os respectivos mecanismos de busca identificados na pesquisa de invenções e criação de inovações frugais.

\begin{tabular}{|l|l|}
\hline Características dos mecanismos de busca & \multicolumn{1}{c|}{ Mecanismos de busca identificados } \\
\hline $\begin{array}{l}\text { Software para gestão de referências } \\
\text { bibliográficas }\end{array}$ & Zotero \\
\hline Pesquisa bibliográfica do Google Acadêmico & Harzing's Publish or Perish \\
\hline Wikipedia como Thesaurus para inovação & $\begin{array}{l}\text { Rede colaborativa de informação e conhecimento } \\
\text { Links cooperativos por categorias: criatividade, } \\
\text { inovação e termoeletricidade }\end{array}$ \\
\hline Patentes como fonte de inovações & $\begin{array}{l}\text { Acesso a bases de patentes WIPO, EPO, WIPO, } \\
\text { Google Patents, USPTO } \\
\text { Instrumentos para análise de patentes }\end{array}$ \\
\hline Solução criativa de problemas & Matriz TRIZ \\
\hline
\end{tabular}

\section{Quadro 2: Modelos de busca de informações}

Fonte: Autores 


\section{CONCLUSÕES E RECOMENDAÇÕES}

A Web 2.0 configura uma fonte inesgotável de informações para o campo da CI com a construção, disseminação e compartilhamento de informações. Seu advento fez surgir mecanismos que permitem a apropriação de dados científicos e tecnológicos. A proposta de método para este estudo utilizou como referência a criação de inovações frugais, adotando como termo de pesquisa o "efeito Peltier". Os mecanismos identificados foram agrupados em cinco grupos e estão alinhados com os fundamentos teóricos relacionados com os objetivos deste estudo.

Resumidamente, o software Zotero pode criar uma base de dados exclusiva para uma pesquisa com links de artigos, imagens, áudios, vídeos e fotos disponibilizados pela Web 2.0. A extensa literatura do Google Acadêmico pode ser acessada por um buscador específico para a elaboração de uma pesquisa bibliográfica. A Wikipedia é um Thesaurus de natureza colaborativa. E as mais importantes bases de patentes mundiais, fontes relevantes para pesquisa de invenções e inovações podem ser acessadas por sites específicos de forma gratuita. Esses quatro mecanismos de busca permitem a apropriação de dados científicos e tecnológicos para utilização na pesquisa de invenções e criação de inovações frugais. Por sua vez, a matriz TRIZ pode contribuir para a melhoria de produtos e desenvolvimento de novos.

O número de informações disponíveis na Web cresce exponencialmente, o que torna mais interessante a utilização dos mecanismos de busca, que também crescem e se aperfeiçoam para atender às novas demandas.

Alguns mecanismos de busca identificados neste estudo estão relacionados com a gestão da informação e do conhecimento e a gestão da inovação, que seriam incluídas, obrigatoriamente, em qualquer processo prático de pesquisa de invenções e criação de inovação. Por isso, é natural o alinhamento dos temas tratados neste estudo, como CI, Web 2.0, inovação frugal e análise das fontes de inovação.

A inserção da Web 2.0 reforça a relação entre a CI e a CC e incentiva a geração de novos mecanismos de divulgação e acesso à informações, aproveitando o maior benefício dessa plataforma, que é a possibilidade de 
ações cooperativas para ampliar o processo interativo entre usuários e a base de dados que cresce exponencialmente. Além disso, a identificação de alguns mecanismos deverá expandir o campo de atuação da CI e tornar-se fator relevante para a pesquisa de invenções e criação de inovações.

A transformação da Web em um cérebro global (Lai \& Turban, 2008, p. 394) leva à exigência de competência e da criatividade como suporte à análise das fontes de inovação para favorecer o desenvolvimento de produtos e seriços que garantam a competitividade da empresa.

Outra constatação pode ser também considerada uma recomendação para próximos estudos: quanto mais a discussão sobre inovação passa a agregar conceitos intangíveis, mais ela se distancia dos aspectos físicos ("hardware") e passa a agregar competências ("software"), invertendo o foco tradicional da gestão de inovação para a inovação de gestão, campo específico das ciências sociais aplicadas, criando inovação organizacional em marketing; em empreendedorismo e em outras áreas.

Sendo assim, o uso criativo de alguns mecanismos pesquisados, como o TRIZ, pode contribuir para o desenvolvimento de processos administrativos em setores de engenharia, bem como em inovações organizacionais.

\section{REFERÊNCIAS}

Abbas, A., Zhang, L., \& Khan, S.U. (2014). A literature review on the stateof-the-art in patent analysis. World Patent Information, 37, 3-13.

Almind, T. C., \& Ingwersen, P. (1997). Informetric analyses on the world wide web: methodological approaches to "webometrics". Journal of Documentation, 53(4), 404-426.

Altshuller, G. S. (1984). Creativity as an exact science - the theory of the solution of inventive problems. Luxembourg: Gordon \& Breach.

Amabile, T. M., Conti, R., Coon, H., Lazenby, J., \& Herron, M. (1996). Assessing the work environment for creativity. Academy of Management Journal, 39(5), 1154-1185.

Basu, R. R., Banerjee, P. M., \& Sweeny, E. (2013). Frugal innovation core competencies to address global sustainability. Journal of Management for Global Sustainability, 2, 63-82. 
Bharadwaj, S., \& Menon, A. (2000). Making innovation happen in organizations: Individual mechanisms, organizational creativity mechanisms or both? Journal of Product Innovation Management, 17, 424-434.

Bhatti, Y. (2012). What is frugal, what is innovation? Towards a theory of frugal innovation. Social Science Research Network. Recuperado em 20 de dezembro, 2014 de http://papers.ssrn.com/sol3/papers.cfm?abstract_id=2005910

Blau, J. R, \& McKinley, W. (1979). Ideas, complexity, and innovation. Administrative Science Quarterly, 24, 200-219.

Bush, V. (1945). As we may may think. Atlantic Monthly, 176(1), 101-108.

Cendon, B. V. (2001, janeiro/abril). Ferramentas de busca na Web. CI Inf., $30(1), 39-49$.

European Commission (1995). Green paper on innovation. Recuperado em 13 de março, 2011, de http://europa.eu/documents/comm/green_papers/pdf/com95_688_en.p df

Eisenhardt, K. M. (1989, October). Building theories from case study research. Academy of Management Review, 14(4), 532-550.

Espacenet. European Patent Office. (n.d.). Recuperado em 9/12/2-14 de http://worldwide.espacenet.com.

Feldman, L. B., Ruthes, R. M., \& Cunha, I.C.K.O. (2008, março-abril). Criatividade e inovação: competências na gestão de enfermagem. Revista Brasileira de Enfermagem, 61(2), 239-242.

Ferreira, A. A., Guimarães, E. R., \& Contador, J. C. (2009). Patente como instrumento competitivo e como fonte de informação tecnológica. Gest. Prod., 16(2), 209-221.

Fleury, M. T. L., \& Fleury, A. (2001). Construindo o conceito de competência. $R A C$, Edição Especial, 183-196.

Google Patents. (n.d.) Recuperado em 9 de dezembro, 2014, de http://www.google.com/?tbm=pts

Lai, L. S. L., \& Turban, E. (2008). Groups formation and operations in the Web 2.0. Environment and social networks. Group Decis Negot, 17(5), 387-402.

Macia-Chapula, C. A. (1998, maio/agosto). O papel da informetria e da cienciometria e sua perspectiva nacional e internacional. Ciência da Informação, 27(2), 134-140.

Mooers, C. N. (1951). Zatocoding applied to mechanical organization of knowledge. American Documentation, 2, 20-32. 
Moura, M. A. (2009). Informação, ferramentas ontológicas e redes sociais ad hoc: a interoperabilidade na construção de tesauros e ontologias. Informação \& Sociedade. Estudos, 19(1), 59-74.

O'Reilly, T. (2005). What is Web 2.0 design patterns and business models for the next generation of software. Recuperado em 20 de dezembro, 2014, de http://www.oreilly.com/pub/a/web2/archive/what-is-web-20.html.

Patentscope. (n.d.). World Intellectual Property Organization. Recuperado em 9 de setembro, 2014, de http://www.wipo.int/patentscope/search/en/search.jsf.

Plonski, G. A. (2005, janeiro/março). Bases para um movimento pela inovação tecnológica no Brasil. São Paulo em Perspectiva, 19(1), 25-33.

Prahalad, C. K., \& Hart, S. L. (2002, January). The fortune at the bottom of the pyramid. Strategy Business, 26, 54-67.

Puccio, G. J. \& Cabra J. F. (2010). Organizational creativity: a systems approach. In J. Kaufmann, \& R. J. Sternberg (Eds.), The Cambridge handbook of creativity (pp. 145-173). New York, NY: Cambridge University Press.

Ruas, R. (2005). Literatura, dramatização e formação gerencial: a apropriação de práticas teatrais ao desenvolvimento de competências gerenciais. Organizações \& Sociedade, 12(32), 121-142.

Sachs, I. (2004). Desenvolvimento: includente, sustentável e sustentado. Rio de Janeiro: Garamond.

Sachs, I. (2010). Barricadas de ontem,campos de futuro. Estudos avançados. 24(68), 25-38.

Saracevic, T. (1996). Ciência da informação: origem, evolução e relações. Perspectivas em Ciência da Informação, 1(1). Recuperado em 18 de dezembro, 2014 http://portaldeperiodicos.eci.ufmg.br/index.php/pci/article/view/235

Soo, C., Devinney, T., Midgley, D., \& Deering, A. (2002). Knowledge management: Philosophy, processes, and pitfalls. California Management Review, 44, 129-150.

Strong, M. (1993). Prefácio. In I. Sachs, Estratégia de transição para o século $X X I$. São Paulo: Fundap.

Valentim, M. L. P. (2008). Criatividade e inovação na atuação profissional. CRB-8 Digital, 1(1), 3-9.

Vanti, N. A. P. (2002, maio/agosto). Da bibliometria à webometria: uma exploração conceitual dos mecanismos utilizados para medir o registro da informação e a difusão do conhecimento. Ciência da Informação, 31(2), 152-162. 
Wikipedia. (n.d.). Recuperado em 10 de dezembro, 2014, de http://en.wikipedia.org/wiki/Main_Page.

Wormell, I. (1998). Informetria: explorando bases de dados como instrumentos de análise. Ciência da Informação, 27(2).

Yin, R. (2010). Estudo de caso - planejamento e métodos (4a. ed.). Porto Alegre: Bookman.

Zeschky, M. B., Winterhalter, S., \& Gassmann, O. (2014). From cost to frugal and reverse innovation: mapping the field and iImplications for global competitiveness. 